\title{
Deregulation of the G1/S phase transition in cancer and squamous intraepithelial lesions of the uterine cervix: A case control study
}

\author{
DEMETRIOS A. ARVANITIS ${ }^{1}$ and DEMETRIOS A. SPANDIDOS ${ }^{2}$ \\ ${ }^{1}$ Molecular Biology Division, Biomedical Research Foundation, Academy of Athens, Athens; \\ ${ }^{2}$ Department of Virology, Medical School, University of Crete, P.O. Box 1393, Heraklion, Crete, Greece
}

Received July 23, 2008; Accepted August 25, 2008

DOI: 10.3892/or_00000070

\begin{abstract}
High-risk types of HPV express the oncoproteins, E6 and E7, that can inactivate TP53 and RB1, respectively, and thus take control of both cell cycle and apoptosis. Herein, the mRNA expression profiles of $24 \mathrm{G} 1 / \mathrm{S}$ checkpoint genes were analysed in cancer and squamous intraepithelial lesions (SIL) of the uterine cervix. In total 35 squamous cervical carcinomas, 26 high-grade SIL (HSIL), 33 low-grade SIL (LSIL) tissues, and 28 normal uterine cervix specimens as controls were assessed by RT-PCR. Five genes were found to be upregulated only in tumours, RBL2, E2F2, CDK6, CCNE1 and MYC; eight in tumours and HSILs, E2F1, E2F3, E2F5, CCND1, CDK2, CDKN1B, PCNA and POLA, and five in tumours, HSILs and LSILs, TP53, E2F4, CDKN1A, CDKN2A and DHFR. MDM2 was found to be upregulated in SIL, while RBL1 was found to be downregulated in all three groups of cases. TP73 exhibited lower levels in carcinomas; however, its exon 13-containing isoforms were increased and exon 2-containing isoforms were reduced in both cancer and HSIL. Three genes, RB1, CDK4 and CDKN2D, did not exhibit any significant alteration in gene expression. Hierarchical clustering revealed that this set of $\mathrm{G} 1 / \mathrm{S}$ checkpoint genes was able to discriminate the total 122 samples into groups of disease and non-disease with only 8 exceptions (6.6\%). Our data suggest that deregulation of $\mathrm{G} 1 / \mathrm{S}$ phase transition in cervical carcinogenesis is a progressive process. Certain clusters of genes are activated very early in pre-cancerous SILs while others are activated later, during malignant transformation. The ability of this array of markers to identify disease status suggests that it could be used for diagnostic purposes.
\end{abstract}

Correspondence to: Professsor D.A. Spandidos, Department of Virology, Medical School, University of Crete, P.O. Box 1393, Heraklion, Crete, Greece

E-mail: spandidos@spandidos.gr

Key words: cyclin, CDK, CDKN, TP53, TP73, retinoblastoma, pocket proteins

\section{Introduction}

Cervical cancer is the second most common gynaecological malignancy worldwide, with $\sim 500,000$ new cases and 274,000 deaths per annum. It is much more common in the developing countries, where $83 \%$ of cases occur (1). Chronic infection of keratinocytes of the uterine cervix by certain types of human papillomavirus (HPV), classified as highrisk, is well established as the causative agent of both invasive cervical cancer and cervical intraepithelial neoplasia $(2,3)$. Despite the development of an HPV vaccine for certain high-risk types (4-6) and the development of national HPV vaccination policies, a large number of the female population, remains at risk (7).

Carcinogenesis of the uterine cervix, from HPV infection to viral persistence, to the development of precancerous lesions, and finally invasive cancer, is a complex process that remains to be elucidated. Cervical carcinoma develops infrequently, even after infection with high-risk types of HPV, and it typically occurs years to decades after the initial infection (8). The cancer precursors, squamous intraepithelial lesions (SILs) are also infected by high-risk HPV types but do not exhibit such a proliferative or invasive phenotype as tumours (9). Thus, the development of new biomarkers that could accurately characterize each step of this process is important for the better clinical management of women suffering HPV infection.

Unlike the HPV-associated benign lesions, where the viral genome is maintained episomally, the majority of HPVassociated cancers contain viral DNA integrated into the genome of the host cells $(10,11)$. While most of the open reading frames of the integrated viral DNA are lost or interrupted, the HPV oncoproteins, E6 and E7, continue to be expressed (10). Furthermore, the expression of both proteins is upregulated upon integration, because the gene encoding the viral transcriptional repressor E2 is generally lost and the mRNA encoding E6 and E7 is stabilized (12,13). E6 and E7 proteins encoded by high-risk types of HPV interact with TP53 and the retinoblastoma protein (RB1) respectively to inactivate these tumour suppressors (14-16).

Progression through the cell cycle depends on the coordinated synthesis, activation, and degradation of a family of cyclins (CCNs) that act as catalytic subunits of 
cyclin-dependent kinases (CDKs). Different CCN/CDK holoenzymes are activated at specific phases of the cell cycle and exert their regulatory control by phosphorylating key proteins, such as transcription factors, involved in cell cycle progression (17). The activity of these kinases is directed in part by inhibitors of CDKs (CDKNs). Two classes of CDKN exist; the Cip/Kip family, which includes CDKN1A (p21), CDKN1B (p27) and CDKN1C (p57), CDKNs that regulate cell proliferation throughout the cell cycle; and the INK4 proteins, CDKN2B (p15), CDKN2A (p16), CDKN2C (p18) and CDKN2D (p19), which are inhibitors of CDK4 and CDK6 and are active only at G1 phase $(18,19)$.

The RB1 protein is also an important negative regulator of proliferation that, during the resting G0 state, is unphosphorylated and inhibits cell cycle progression. During G1 to S phase transition, when it is hypophosphorylated, it binds to the transcription factor, E2F, and prevents the transcription of genes involved in the transition, such as polymerase A (POLA), dihydrofolate reductase (DHFR), MYC and proliferating cell nuclear antigen (PCNA); and, when it is phosphorylated by CCND1/CDK4/6, it permits cells to traverse the G1/S checkpoint and proliferate (20). The Rb-like RBL1 (p107) and RBL2 (p130), also known as pocket proteins, display similar and distinct functions to RB1 in G1/S transition control, but they contain a Cip/Kip like motif which enables binding and inhibition of CDKs (20).

The E2F family of transcription factors plays an important role in the control of the cell cycle and action of tumour suppressor proteins. The E2F proteins contain conserved domains, including a DNA-binding domain, a dimerization domain which determines interaction with the differentiationregulated transcription factor proteins (DP), a transactivation domain enriched in acidic amino acids, and a tumour suppressor protein association domain which is embedded within the transactivation domain. E2F1, E2F2 and E2F3 have an additional cyclin-binding domain, while E2F4 binds preferentially to RBL1/RBL2 and E2F5 to $\operatorname{RBL} 2(21,22)$.

The TP53 protein regulates, as transcriptional factor, the expression of CDKN1A, which acts as a universal inhibitor, able to bind to and inhibit CDKs at all stages of the cell cycle. When it is overexpressed, CDKN1A inhibits CCND1/CDK4/6 kinase activity and arrests cells in G1 (23). TP53 can be induced in response to DNA damage and could be degraded by the action of the MDM2 protein, while CDKN2D counteracts this effect (24). TP73, one of the two TP53 homologues, can be divided similarly to $\mathrm{p} 53$ functional domains, into amino-terminal transactivation, central DNA-binding domain and homo-oligomerization domain. However, it harbours an extended, compared with TP53, carboxy-terminal where two additional domains exist, a secondary transactivation and a sterile alpha motif (SAM) domain (25). It shares overlapping functions with TP53 to some extent, such as CDKN1A and MDM2 transcriptional activation, but has a unique role in TP53-independent apoptosis through E2F1 (26). The various TP73 isoforms, those with truncated carboxy-terminal (exon 13 lacking) and others with truncated amino-terminal (exon 2 lacking) have different transactivation efficacies and are able to hetero-oligomerize with TP53 mutants to form tetramers with variable ability to activate and antagonise for TP53responsive promoters (25).
Table I. Oligonucleotide primer sequences.

\begin{tabular}{llc}
\hline Primers & \multicolumn{1}{c}{ Sequence (5'-3') } & $\begin{array}{c}\text { Fragment } \\
(\mathrm{bp})\end{array}$ \\
\hline E2F1-F & GTGCAGATTGGAGGGTGG & 154 \\
E2F1-R & GATGGATATGAGATGGGAGAGG &
\end{tabular}

E2F2-F CCTTGGAGGCTACTGACAGC

E2F2-R CCACAGGTAGTCGTCCTGGT

E2F3-F CACGTACCCCGTAGGAAAAG

E2F3-R TCAGTGTGTGCATGACAACTC

E2F4-F TTTTATTCACTTGAACACTGTAC

E2F4-R CAGAGATTTAGAAAGATTTACAG

E2F5-F TTTAATATCCACAAACGTCCCC

E2F5-R GGCCACTGTTTTGATGACCT

RB1-F TGGGTGATTCCTAAGCCACTTGA

RB1-R GGACTCCCACTCTAGGGCCATT

RBL1-F TGTGGTGAGCCAAGGTTG RBL1-R TGGGGTTTCTCCTTATTTCA

RBL2-F AGCAGCAGCCTTTAATGCAT

RBL2-R CTTGCAGTATTTCTAAAAGCTTTGC

POLA-F TTGCCCCTTAAATAGAAAGTGC 215

POLA-R TTTTGCTGCTGCCAAAGAC

B2M-F TCCAACATCAACATCTTGGT

B2M-R TCCCCCAAATTCTAAGCAGA

CCND1-F AAAGACAGTTTTTGGGTAATCTTTT 126

CCND1-R CCGGAGCATTTTGATACCAG

CCNE1-F ACAAAACAGGTTCATCAAAGGC

CCNE1-R GGTGCTACTTGACCCTAAGGG

CDK2-F TTCTTGAAGCCCCCAGC

CDK2-R CCCCTGTATTCCCAGAGTTG

CDK4-F CTTCTGGACACTGAGAGGGC

CDK4-R TGGGAGGGGAATGTCATTAA

CDK6-F CGGAGAACACCCTTGGTG

CDK6-R GAGCCTGTCCAGAAGACAGC

PCNA-F ACTCGCATTTAATGATGGTG

PCNA-R CTTCTTTCATGAAGCAGTGG

MYC-F GGAAAAGTAAGGAAAACGATTCC

MYC-R TAGGATTGAAATTCTGTGTAACTGC

DHFR-F ACCAACATGTGAAAAGCCCG

DHFR-R ACCTGCTACAGTGAGCTGCC

MDM2-F TGAAGGTTTCTCTTCCTGAAGC

MDM2-R AAGGTGGGAGTGATCAAAAGG

TP53-F TTTGGGTCTTTGAACCCTTG

TP53-R CCACAACAAAACACCAGTGC

CDKN1A-F ATTCAGCATTGTGGGAGGAG CDKN1A-R TGGACTGTTTTCTCTCGGCT 
Table I. Continued.

\begin{tabular}{llc}
\hline Primers & \multicolumn{1}{c}{ Sequence (5'-3') } & $\begin{array}{c}\text { Fragment } \\
(\mathrm{bp})\end{array}$ \\
\hline CDKN2A-F & CATCAGTCACCGAAGGTCCT & 150 \\
CDKN2A-R & AATGGACATTTACGGTAGTGGG & \\
CDKN2D-F & GATCTGGGGTCACCCTCTC & 207 \\
CDKN2D-R & CCAACACACCAAAAGGAGTG & \\
TP73-exon2-F & CACCACGTTTGAGCACCTC & 94 \\
TP73-exon2-R & CGCCCACCACCTCATTATT & \\
TP73-F & GGACGTCTTCCACCTGGAG & 216 \\
TP73-R & GTAGTCGGTGTTGGAGGGG & \\
TP73-exon13-F & TTTTAACAGGATTGGGGTG & 91 \\
TP73-exon13-R & CTCAATGGTCAGGTTCTGC & \\
\hline
\end{tabular}

The transition of the cell cycle from G1 to $\mathrm{S}$ phase requires both cyclin D-dependent $\mathrm{Cdk} 4$ and $\mathrm{Cdk} 6$, and cyclin $\mathrm{E} / \mathrm{Cdk} 2$ holoenzymes, and is regulated by the TP53/MDM2/ CDKN1A/CDKN2D and RB1/E2F/CCND1/CDK4/CDK6/ CDKN2A pathways $(23,27)$. Inactivation of TP53 and pocket proteins, RB1, RBL1 and RBL2, by the viral oncoproteins of high-risk HPV types, E6 and E7, is a prerequisite for oncogenesis but is not sufficient to convert normal cells into an immortalized or malignant state (2). A number of secondary factors, such as smoking, multiparity and oral contraceptives, are thought to influence the likelihood that a high-risk type HPV infection will persist and progress to cervical cancer, possibly though genetic and epigenetic alterations, activation of proto-oncogenes and loss of tumour suppressors as well as immunological responses $(28,29)$.

In order to investigate the potential contribution to human cervical carcinogenesis of the genes implicated in G1/S phase transition, and to identify possibly useful biomarkers for HPV infection progression, the aim of this study was to determine and analyse the transcriptional levels of RB1, RBL1, RBL2, TP53, TP73 and its isoforms, MDM2, E2F1, E2F2, E2F3, E2F4, E2F5, CCND1, CCNE1, CDK2, CDK4, CDK6, CDKN1A, CDKN1B, CDKN2A, CDKN2D, DHFR, MYC, PCNA, and POLA in cervical cancer, SIL and the normal uterine cervix.

\section{Materials and methods}

Specimen collection. Thirty-five squamous cervical carcinomas, staged between IA and IIB according to the International Federation of Gynaecology and Obstetrics (FIGO) and World Health Organization (WHO) systems; 26 high-grade SIL (HSIL) and 33 low-grade SIL (LSIL) tissues, classified according to the Bethesda system; and 28 normal uterine cervix specimens, were obtained from 122 women aged 25-60 (40.6 \pm 7.9$)$ years, at the Department of Obstetrics and Gynaecology of the University Hospital of Heraklion, Crete. Disease status was determined by cytological, colposcopy and biopsy examination. All tissue samples were snap frozen, stored at $-80^{\circ} \mathrm{C}$ and tested for HPV DNA, using the $\mathrm{GP}^{+} / 6^{+}$primer set $(30)$. The positive samples were further investigated using specific primers, previously described (31), for HPV-16, -18, -33 and -11 , in multiplex PCR reactions with B2-microglobulin (B2M) as internal control. The University of Crete ethics committee approved this study and all the participants gave written informed consent.

$R N A$ preparation and $R T-P C R$. Total RNA was extracted from cervical epithelium tissues that were separated from adjacent stromal tissue by microdissection, using the Trizol reagent (Invitrogen Corp, Carlsbad, CA, USA) according to the manufacturer's instructions. RNA quality was estimated by agarose electrophoresis, while its concentration and purity was determined by UV spectrophotometry. Using Thermoscript reverse transcriptase (Invitrogen Corp.) cDNA was prepared from $3 \mu \mathrm{g}$ of total RNA followed by multiplex PCR amplification with Platinum Taq DNA polymerase (Invitrogen Corp.) and the appropriate primer sets (Table I). In each multiplex PCR assay, the B2-microglobulin (B2M) target cDNA was amplified as reference. The RT-PCR products were analyzed on $8 \%$ polyacrylamide gels (29:1 ratio acrylamide/bis-acrylamide), in $0.5 \mathrm{X}$ Tris-Boric-EDTA (TBE) buffer and silver-stained. The analysis was repeated 3 times for each sample and marker, and the results were reproducible. Integrated density (ID) of the bands was calculated by digital imaging using the Image J 1.32 j software (Wayne Rasband, National Institutes of Health, USA, http://rsb.info.nih gov/ij/), as ID=[mean density-background]*pixels. These measurements were used as a quantitative parameter for gene expression.

Statistics. Descriptive statistics, calculations of mean, standard deviation (SD), standard error (SE), KolmogorovSmirnov goodness of fit and Levene median test, for the examination of normality and equivalency of variances respectively, were performed for each marker and group examined. Evaluation of the power of this experimental protocol to identify differences of gene expression was tested by multiple comparison analysis of the reference gene B2M expression from independent RT-PCR reactions of the same sample. Student's t-test, 2-tailed, was executed and a $99 \%$ confidence interval was determined between 0.82 and 1.20 $(\mathrm{p}<0.01)$. Thus, a ratio of average gene expression levels between case and control group $<0.82$ was considered as downregulation, while a ratio $>1.20$ was considered as upregulation. Kruskal-Wallis one-way analysis of variance on ranks, a nonparametric test, was used to compare the expression levels of each marker between different groups Another nonparametric test, the Spearman rank order correlation, was performed to investigate correlations of expression among genes. Classification of samples according to gene expression was performed by hierarchical clustering using calculation of Euclidean distance. The $99 \%$ reference range for the mean was constructed conservatively as mean $\pm 3 \times$ SD. A p-value $<0.05$ was considered statistically significant. All statistical calculations were carried out by the SigmaStat 3.00 (SPSS Inc., USA) software except hierarchical clustering which was performed by SYSTAT 10 (SPSS Inc.). 
Table II. Statistically significant correlation coefficient (CC) values calculated for pairs of genes.

\begin{tabular}{|c|c|c|c|c|c|c|c|c|}
\hline A) & CDK2 & CDKN2D & MYC & 3CDKN1A & CDKN2A & MDM2 & RBL2 & CDK4 \\
\hline E2F4 & 0.728 & & 0.602 & 0.634 & 0.679 & 0.134 & 0.527 & 0.372 \\
\hline CDK2 & & & 0.529 & 0.574 & 0.628 & 0.147 & 0.507 & 0.320 \\
\hline \multicolumn{9}{|l|}{ CDKN2D } \\
\hline MYC & & & & 0.618 & 0.595 & & 0.325 & \\
\hline CDKN1A & & & & & 0.588 & 0.359 & 0.447 & 0.245 \\
\hline CDKN2A & & & & & & & 0.509 & \\
\hline MDM2 & & & & & & & 0.244 & 0.192 \\
\hline RBL2 & & & & & & & & 0.222 \\
\hline \multicolumn{9}{|l|}{ CDK4 } \\
\hline \multicolumn{9}{|l|}{ RBL1 } \\
\hline \multicolumn{9}{|l|}{ PCNA } \\
\hline \multicolumn{9}{|l|}{ CDKN1B } \\
\hline \multicolumn{9}{|l|}{ CDK6 } \\
\hline \multicolumn{9}{|l|}{ E2F2 } \\
\hline \multicolumn{9}{|l|}{ RB1 } \\
\hline \multicolumn{9}{|l|}{ CCNE1 } \\
\hline \multicolumn{9}{|l|}{ DHFR } \\
\hline \multicolumn{9}{|l|}{ E2F5 } \\
\hline \multicolumn{9}{|l|}{ E2F1 } \\
\hline \multicolumn{9}{|l|}{ POLA } \\
\hline \multicolumn{9}{|l|}{ TP53 } \\
\hline \multicolumn{9}{|l|}{ TP73 } \\
\hline \multicolumn{9}{|l|}{ E2F3 } \\
\hline \multicolumn{9}{|l|}{ TP73 exon13 } \\
\hline CCND1 & & & & & & & & \\
\hline
\end{tabular}

\begin{tabular}{|c|c|c|c|c|c|c|c|c|}
\hline B) & RBL1 & PCNA & CDKN1B & CDK6 & $\mathrm{E} 2 \mathrm{~F} 2$ & RB1 & CCNE1 & DHFR \\
\hline E2F4 & -0.793 & 0.757 & 0.759 & 0.460 & 0.557 & 0.337 & 0.424 & 0.765 \\
\hline CDK2 & -0.744 & 0.688 & 0.693 & 0.485 & 0.491 & 0.316 & 0.452 & 0.734 \\
\hline \multicolumn{9}{|l|}{ CDKN2D } \\
\hline MYC & -0.651 & 0.606 & 0.574 & 0.479 & 0.483 & 0.241 & 0.274 & 0.586 \\
\hline CDKN1A & -0.624 & 0.578 & 0.600 & 0.437 & 0.375 & 0.231 & 0.292 & 0.642 \\
\hline CDKN2A & -0.719 & 0.686 & 0.666 & 0.558 & 0.440 & 0.318 & 0.437 & 0.708 \\
\hline MDM2 & & & & & & -0.300 & & \\
\hline RBL2 & -0.464 & 0.476 & 0.531 & 0.228 & 0.328 & 0.253 & 0.412 & 0.458 \\
\hline CDK4 & -0.279 & 0.282 & 0.302 & & 0.189 & 0.146 & & 0.377 \\
\hline RBL1 & & -0.722 & -0.755 & -0.594 & -0.589 & -0.386 & -0.504 & -0.800 \\
\hline PCNA & & & 0.812 & 0.463 & 0.511 & 0.397 & 0.445 & 0.722 \\
\hline CDKN1B & & & & 0.526 & 0.505 & 0.486 & 0.582 & 0.714 \\
\hline CDK6 & & & & & 0.391 & 0.272 & 0.386 & 0.557 \\
\hline $\mathrm{E} 2 \mathrm{~F} 2$ & & & & & & 0.298 & 0.422 & 0.574 \\
\hline RB1 & & & & & & & 0.437 & 0.405 \\
\hline CCNE1 & & & & & & & & 0.429 \\
\hline \multicolumn{9}{|l|}{ DHFR } \\
\hline \multicolumn{9}{|l|}{ E2F5 } \\
\hline \multicolumn{9}{|l|}{ E2F1 } \\
\hline \multicolumn{9}{|l|}{ POLA } \\
\hline \multicolumn{9}{|l|}{ TP53 } \\
\hline \multicolumn{9}{|l|}{ TP73 } \\
\hline \multicolumn{9}{|l|}{ E2F3 } \\
\hline \multicolumn{9}{|l|}{ TP73 exon13 } \\
\hline CCND1 & & & & & & & & \\
\hline
\end{tabular}


Table II. Continued.

\begin{tabular}{|c|c|c|c|c|c|c|c|c|c|}
\hline C) & E2F5 & E2F1 & POLA & TP53 & TP73 & E2F3 & TP73 exon 13 & CCND1 & TP73 exon 2 \\
\hline E2F4 & 0.647 & 0.747 & 0.698 & 0.676 & -0.475 & 0.602 & 0.677 & 0.723 & -0.726 \\
\hline CDK2 & 0.576 & 0.619 & 0.613 & 0.678 & -0.440 & 0.568 & 0.625 & 0.702 & -0.657 \\
\hline \multicolumn{10}{|l|}{ CDKN2D } \\
\hline MYC & 0.511 & 0.552 & 0.526 & 0.529 & -0.463 & 0.535 & 0.485 & 0.542 & -0.568 \\
\hline CDKN1A & 0.505 & 0.551 & 0.563 & 0.551 & -0.353 & 0.532 & 0.487 & 0.533 & -0.540 \\
\hline CDKN2A & 0.528 & 0.658 & 0.559 & 0.620 & -0.406 & 0.557 & 0.501 & 0.572 & -0.598 \\
\hline MDM2 & 0.228 & & & & & & & & \\
\hline RBL2 & 0.404 & 0.450 & 0.410 & 0.476 & -0.261 & 0.458 & 0.337 & 0.418 & -0.405 \\
\hline CDK4 & 0.387 & 0.289 & 0.321 & 0.430 & -0.334 & 0.213 & 0.366 & 0.381 & -0.364 \\
\hline RBL1 & -0.660 & -0.767 & -0.703 & -0.751 & 0.549 & -0.662 & -0.677 & -0.767 & 0.782 \\
\hline PCNA & 0.604 & 0.600 & 0.667 & 0.668 & -0.515 & 0.604 & 0.680 & 0.683 & -0.687 \\
\hline CDKN1B & 0.615 & 0.667 & 0.682 & 0.647 & -0.509 & 0.613 & 0.664 & 0.647 & -0.674 \\
\hline CDK6 & 0.412 & 0.552 & 0.418 & 0.471 & -0.353 & 0.461 & 0.478 & 0.485 & -0.469 \\
\hline E2F2 & 0.495 & 0.548 & 0.537 & 0.525 & -0.428 & 0.422 & 0.538 & 0.484 & -0.460 \\
\hline RB1 & 0.234 & 0.378 & 0.324 & 0.379 & -0.383 & 0.317 & 0.339 & 0.290 & -0.367 \\
\hline CCNE1 & 0.391 & 0.377 & 0.471 & 0.521 & -0.422 & 0.422 & 0.447 & 0.407 & -0.464 \\
\hline DHFR & 0.712 & 0.784 & 0.684 & 0.716 & -0.534 & 0.555 & 0.687 & 0.743 & -0.682 \\
\hline E2F5 & & 0.674 & 0.653 & 0.650 & -0.498 & 0.483 & 0.546 & 0.667 & -0.603 \\
\hline E2F1 & & & 0.666 & 0.578 & -0.475 & 0.499 & 0.583 & 0.719 & -0.644 \\
\hline POLA & & & & 0.675 & -0.509 & 0.506 & 0.706 & 0.709 & -0.646 \\
\hline TP53 & & & & & -0.650 & 0.590 & 0.622 & 0.700 & -0.670 \\
\hline TP73 & & & & & & -0.512 & -0.497 & -0.504 & 0.630 \\
\hline E2F3 & & & & & & & 0.508 & 0.510 & -0.593 \\
\hline TP73 exon13 & & & & & & & & 0.592 & -0.620 \\
\hline CCND1 & & & & & & & & & -0.721 \\
\hline
\end{tabular}

\section{Results}

Five genes, RBL2, E2F2, CDK6, CCNE1 and MYC, were found to be upregulated only in cervical cancer, in 80, 80, 97, 74 and $74 \%$ of cases, respectively. Eight genes were upregulated in both cancer and HSIL: E2F1 in 84 and $71 \%$ of cervical carcinomas and HSILs, respectively; E2F3 in 81 and $74 \%$, E2F5 in 86 and $81 \%$, CCND1 in 77 and 52\%, CDK2 in 88 and $62 \%$, CDKN1B in 86 and 77\%, PCNA in 89 and $69 \%$, and POLA in 62 and 52\%. Only 5 out of the 24 tested genes were found to be upregulated compared to the controls in all three groups of cases, cervical cancer, HSIL and LSIL. TP53 was upregulated in $80 \%$ of tumours, $69 \%$ of HSILs and $64 \%$ of LSILs; E2F4 in 89, 81 and 76\%; CDKN1A in 82, 73 and $70 \%$; CDKN2A in 86,82 and $77 \%$; and DHFR in 75, 70 and $62 \%$, respectively. MDM2 exhibits a unique pattern of upregulation in 88 and $73 \%$ of HSILs and LSILs, respectively.

RBL1 was found to be downregulated in 97,88 and $73 \%$ of tumours, HSILs and LSILs. TP73 exhibited lower levels in $60 \%$ of cervical cancers. However, TP73 exon 13-containing isoforms were substantially increased in 73 and $69 \%$ of cancers and HSILs, while exon 2-containing isoforms were reduced in 97 and $73 \%$ of tumours and HSILs, respectively. Three genes, RB1, CDK4 and CDKN2D, exhibited approximately the same expression levels between cases and controls. All the observed differences of gene expression were statistically significant $(\mathrm{p}<0.05)$ as tested by Kruskal-Wallis one-way analysis of variance on ranks. Representative examples of the RT-PCR analysis mean ID with SD per gene and group are shown in Fig. 1 and graphically in Fig. 2.

The Spearman correlation coefficient (CC) that is calculated with this test quantifies the strength of the association between the variables and varies between -1 and +1 . A CC near +1 indicates there is a strong positive relationship between the two variables, with both increasing together. A CC near -1 indicates there is a strong negative relationship between the two variables, with one always decreasing as the other increases. A CC of 0 indicates no relationship between the two variables. Spearman rank order correlation test was performed and significant correlations $(\mathrm{p}<0.0001)$ between pairs of tested genes were observed (Table II). The highest positive CC significant values were calculated for the pairs; PCNA-CDKN1B (CC: 0.812), DHFR-E2F1 (CC: 0.784), RBL1-TP73 exon 2 (CC: 0.782), E2F4-DHFR (CC: 0.765), E2F4-CDKN1B (CC: 0.759), and E2F4-PCNA (CC: 0.757). The lowest significant negative $\mathrm{CC}$ values were calculated for the pairs; RBL1-DHFR (CC: -0.800), E2F4-RBL1 (CC: -0.793), RBL1-E2F1 (CC: -0.767), RBL1-CCND1 (CC: $-0.767)$, RBL1-CDKN1B (CC: -0.755), and RBL1-TP53 (CC: -0.751).

Nearly all the cervical carcinomas $(94.3 \%)$ were found to be HPV positive, while the vast majority of HSILs and LSILs 


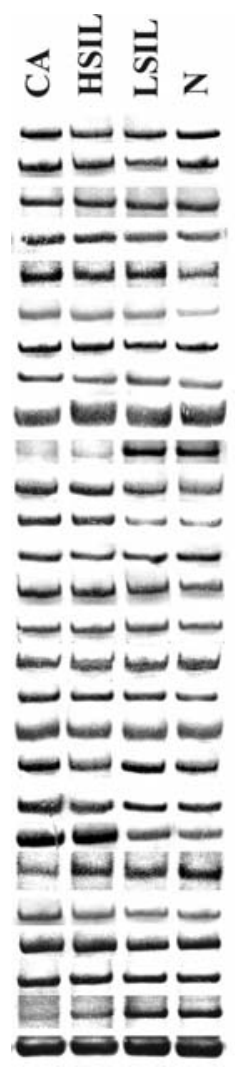

\begin{tabular}{ccccc} 
& \multicolumn{4}{c}{ ID $\left(\mathbf{x 1 0}^{4}\right)[$ Mean \pm SD] } \\
E2F4 & CA & HSIL & LSIL & N \\
CDK2 & $8 \pm 2$ & $7 \pm 1$ & $5 \pm 1$ & $3 \pm 1$ \\
CDKN2D & $8 \pm 3$ & $8 \pm 1$ & $5 \pm 1$ & $4 \pm 1$ \\
MYC & $10 \pm 2$ & $7 \pm 1$ & $7 \pm 2$ & $6 \pm 1$ \\
CDKN1A & $9 \pm 2$ & $8 \pm 1$ & $7 \pm 1$ & $5 \pm 1$ \\
CDKN2A & $13 \pm 2$ & $10 \pm 2$ & $9 \pm 2$ & $6 \pm 3$ \\
MDM2 & $7 \pm 1$ & $9 \pm 2$ & $9 \pm 2$ & $6 \pm 1$ \\
RBL2 & $10 \pm 2$ & $9 \pm 1$ & $9 \pm 2$ & $7 \pm 2$ \\
CDK4 & $14 \pm 1$ & $16 \pm 2$ & $13 \pm 2$ & $13 \pm 2$ \\
RBL1 & $1 \pm 0$ & $4 \pm 1$ & $6 \pm 1$ & $10 \pm 1$ \\
PCNA & $20 \pm 4$ & $14 \pm 3$ & $10 \pm 3$ & $9 \pm 2$ \\
CDKN1B & $19 \pm 3$ & $15 \pm 2$ & $12 \pm 2$ & $10 \pm 3$ \\
CDK6 & $7 \pm 0$ & $6 \pm 2$ & $6 \pm 2$ & $5 \pm 1$ \\
E2F2 & $12 \pm 1$ & $10 \pm 2$ & $8 \pm 3$ & $8 \pm 3$ \\
RB1 & $14 \pm 2$ & $11 \pm 2$ & $10 \pm 2$ & $11 \pm 3$ \\
CCNE1 & $12 \pm 2$ & $10 \pm 2$ & $9 \pm 2$ & $9 \pm 2$ \\
DHFR & $22 \pm 2$ & $20 \pm 2$ & $14 \pm 4$ & $9 \pm 3$ \\
E2F5 & $14 \pm 2$ & $13 \pm 2$ & $10 \pm 2$ & $9 \pm 2$ \\
E2F1 & $14 \pm 1$ & $13 \pm 1$ & $11 \pm 2$ & $9 \pm 2$ \\
POLA & $8 \pm 1$ & $7 \pm 1$ & $5 \pm 2$ & $3 \pm 2$ \\
TP53 & $17 \pm 1$ & $16 \pm 2$ & $12 \pm 3$ & $8 \pm 4$ \\
TP73 & $4 \pm 1$ & $6 \pm 1$ & $7 \pm 2$ & $8 \pm 3$ \\
E2F3 & $16 \pm 2$ & $14 \pm 1$ & $12 \pm 3$ & $10 \pm 3$ \\
TP73 exon13 & $12 \pm 2$ & $11 \pm 1$ & $8 \pm 1$ & $8 \pm 2$ \\
CCND1 & $8 \pm 1$ & $7 \pm 1$ & $5 \pm 1$ & $4 \pm 1$ \\
TP73 exon2 & $1 \pm 1$ & $3 \pm 1$ & $5 \pm 1$ & $6 \pm 1$ \\
B2M & $7 \pm 1$ & $7 \pm 1$ & $8 \pm 1$ & $7 \pm 1$
\end{tabular}

Figure 1. Gene expression analysis. Representative examples of the electrophoresis analysis of RT-PCR products (left panel) and mean ID \pm SD observed in each group. CA, cervical carcinoma; $\mathrm{N}$, normal cervix.

(88.5 and $84.5 \%$, respectively) also harbour the virus. In contrast, all the normal uterine cervix specimens tested were found to be HPV free. The results of genotyping of the HPV positive cases (Table III) shows the predominant presence of HPV-16 in the affected patients. No association were found between gene expression and HPV types.

Hierarchical clustering was performed using calculation of Euclidean distance (Fig. 3). The 122 examined cervical tissue samples were separated into three major clusters (Fig. 3, right panel, sample clustering), normal cervix, LSIL, and cervical cancer together with HSIL. However, the latter cluster
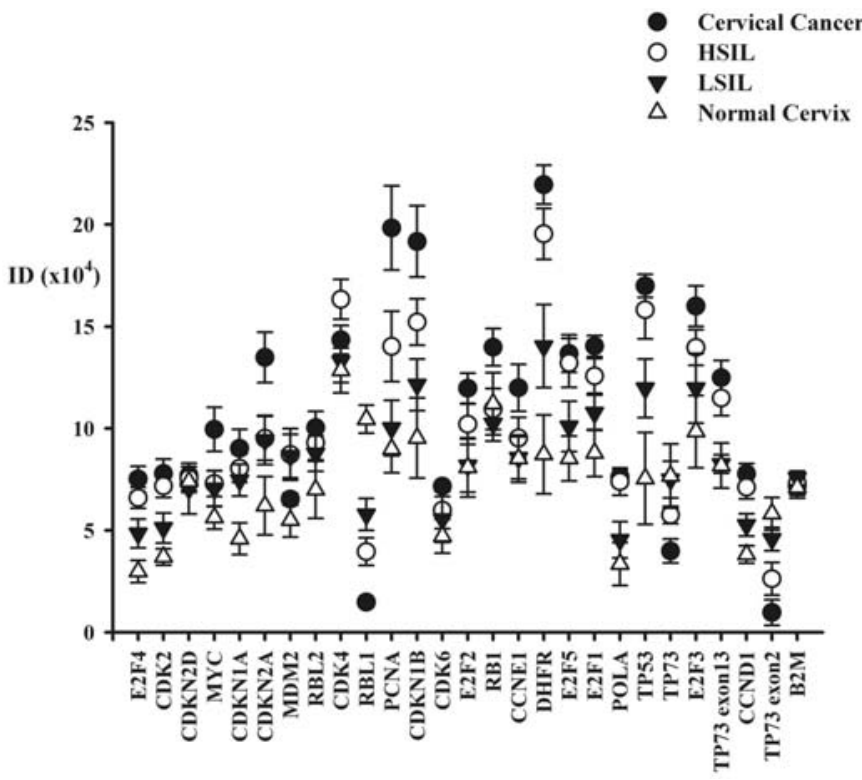

Figure 2. Multiple scatter plot with error bars. Mean ID observed per gene and group is presented. The error bars represent \pm x 3 SD

is clearly separated into two sub-clusters, discriminating HSIL from cancer cases. Only 8 out of 122 samples were incorrectly clustered $(6.6 \%)$. The 24 tested genes were also separated into three clusters (Fig. 3, lower panel, gene clustering). Classification of samples according to gene expression was performed by hierarchical clustering using calculation of Euclidean distance, and a permuted data matrix was designed (Fig. 3). The first contains TP73 exon 2-containing transcripts, RBL1 and TP73 that are expressed in low levels. The second contains POLA, CCND1, CDK2, E2F4, CDK6, MDM2, B2M (reference), CDKN2D, MYC, CDKN1A, RBL2, E2F2, TP73 exon 13-containing transcripts, CCNE1, and CDKN2A, genes with moderate expression. Finally, the third cluster contains CDK4, E2F3, E2F5, E2F1, RB1 PCNA, CDKN1B, TP53 and DHFR that are expressed in high levels.

\section{Discussion}

Deregulation of G1 to S phase transition in cervical carcinogenesis appears to be a progressive process. Initially, after

Table III. Detection of HPV DNA and integration status.

\begin{tabular}{lcccccc}
\hline Specimens & $\begin{array}{c}\text { HPV-16 } \\
(\%)\end{array}$ & $\begin{array}{c}\text { HPV-18 } \\
(\%)\end{array}$ & $\begin{array}{c}\text { HPV-33 } \\
(\%)\end{array}$ & $\begin{array}{c}\text { HPV-11 } \\
(\%)\end{array}$ & $\begin{array}{c}\text { Other HPV } \\
\text { type }(\%)\end{array}$ & $\begin{array}{c}\text { Total HPV } \\
\text { positive (\%) }\end{array}$ \\
\hline Cervical & $19 / 35$ & $5 / 35$ & $3 / 35$ & $0 / 35$ & $6 / 35$ & $33 / 35$ \\
cancer & $(54.3)$ & $(14.3)$ & $(8.6)$ & $(0.0)$ & $(17.1)$ & $(94.3)$ \\
HSIL & $13 / 26$ & $3 / 26$ & $1 / 26$ & $0 / 26$ & $6 / 26$ & $23 / 26$ \\
& $(50.0)$ & $(11.5)$ & $(3.9)$ & $(0.0)$ & $(23.1)$ & $(88.5)$ \\
LSIL & $15 / 33$ & $4 / 33$ & $2 / 33$ & $0 / 33$ & $7 / 33$ & $28 / 33$ \\
& $(45.5)$ & $(12.1)$ & $(6.1)$ & $(0.0)$ & $(21.2)$ & $(84.5)$ \\
\hline
\end{tabular}




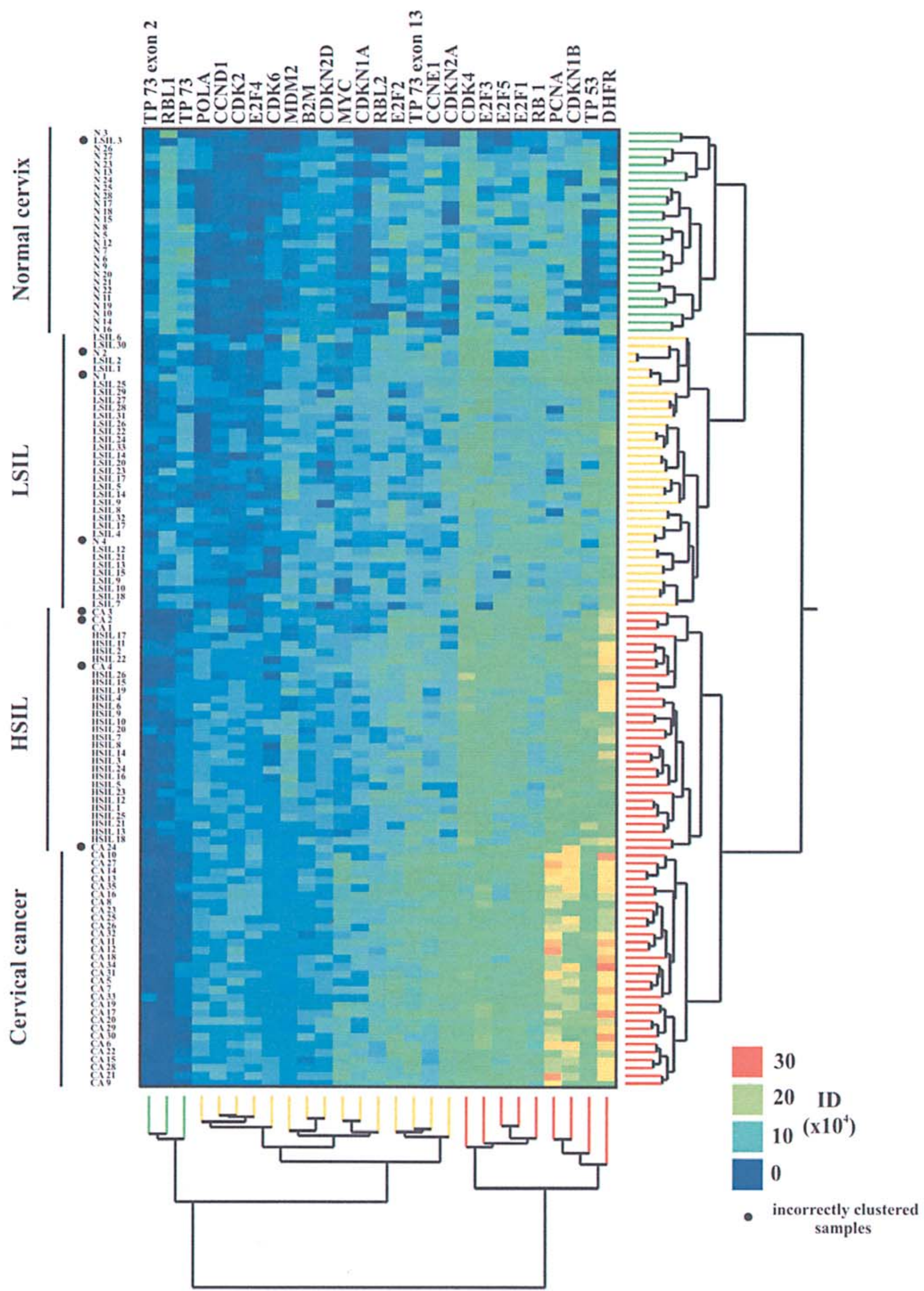

Figure 3. Permuted data matrix. Hierarchical clustering of tested samples and genes. Right panel, sample clustering; lower panel, gene clustering. 
high-risk HPV infection, expression of TP53, MDM2, E2F4, CDKN1A, CDKN2A and DHFR is markedly elevated in LSIL. For RBL1 a decrease in transcription levels was observed in the same set of samples. These alterations that were observed in the majority of LSIL cervical biopsies, when compared with normal cervical tissues, with limited inter-individual variation, affect both TP53 and RB1 pathways.

The TP53 upregulation is probably triggered by episomal HPV presence and then both MDM2 and CDKN1A are also upregulated under the transcriptional activity of TP53 (23). CDKN1A has a major inhibitory effect on the G1/S checkpoint of the cell cycle. The CDKN2A promoter responds to stress signals, limiting cell proliferation and modulating oncogeneinduced apoptosis (24). The increase in DHFR levels, which converts dihydrofolate into tetrahydrofolate, a methyl group shuttle required for the de novo synthesis of purines, necessary for DNA replication, in LSIL suggests deregulation of E2F transcriptional activity (32). RBL1 regulates cell proliferation via phosphorylation-sensitive interactions with E2F transcription factors, and predominantly with E2F4. The combination of RBL1 downregulation and E2F4 upregulation deregulates the ratio of RBL1-E2F4 to RBL2-E2F4 complexes and may alter the transcriptional activity of E2F4 $(33,34)$. Furthermore, the E2F4 and E2F1 transcription factors have opposing roles in cell cycle progression. While E2F1 is an activator of cell cycle progression, E2F4 functions as a transcriptional repressor (35). Thus, overexpression of E2F4 alone deregulates the balance between different activities of E2F factors and might compensate for aggressive cell proliferation.

The findings on gene expression profiles of HSIL cervical biopsies introduce a second group of upregulated genes together with the previously described. This group of genes comprises of E2F1, E2F3, E2F5, CCND1, CDK2, CDKN1B, PCNA and POLA. The TP73 expression remains at the same levels with LSIL and normal cervix. However, its exon 13 containing isoforms are increased while its exon 2 containing isoforms are reduced in HSIL.

E2F1, E2F3, and E2F5 overexpression counteracts the E2F4 upregulation effects in LSIL, and promotes transcriptional activation of the cell cycle progression $\mathrm{S}$ phase genes, such as PCNA and POLA $(35,36)$. CCND1 is one of the main components of $\mathrm{G} 1 / \mathrm{S}$ checkpoint and its upregulation contributes to malignancy (37). Furthermore, CCND1 transcription is regulated by $\mathrm{E} 2 \mathrm{~F}$ (38). CDK2 is a component of the CCNE1/CDK2 complex that along with the CCND1/ CDK4/6 complex are master regulators of progression through G1 phase of the cell cycle (39). CDK2 upregulation promotes G1 to $\mathrm{S}$ phase transition (40). CDKN1B, which is another major inhibitor of the G1/S checkpoint, is possibly overexpressed as a compensatory cellular mechanism to regain normal cell cycle control. Even though TP73 expression is unaltered, the increase of exon 13-containing isoforms together with the decrease of exon 2-containing isoforms suggest that the majority of TP73 isoforms in HSIL cannot surrogate TP53 functions $(23,41,42)$. Our data show that deregulation of $\mathrm{G} 1 / \mathrm{S}$ phase transition is much more severe in HSIL than in LSIL. It is characteristic that, according to gene expression levels, HSIL samples are classified as a subgroup in the same cluster with cervical tumours in hierarchical clustering.
Sequentially, in cervical cancer a third group of genes were found to be upregulated; RBL2, E2F2, CDK6, CCNE1, TP73 and MYC. These alterations in the background of that previously described, with the exception of MDM2, which in turn exhibits normal expression levels, reveal a completely uncontrolled G1/S phase transition. The MDM2 expression levels in cervical cancer might be the outcome of rapid degradation of TP53 through its binding with E6 viral oncoprotein, since MDM2 transcription is controlled by TP53 in a feedback regulation mechanism. The TP73 downregulation effect is further contributing to the disease phenotype together with its isoform equilibrium imbalance.

In interpreting our gene expression data, it is assumed that protein levels in these tissue samples reflect the expression of their corresponding mRNAs. Thus, we generally assume that samples with high mRNA content for a particular gene are likely to have elevated levels for the corresponding protein. We acknowledge that this need not be the case, as posttranscriptional, translational and protein degradation controls probably have a significant influence. However, the limited amounts of biopsy material available have precluded analysis at the protein level.

Spearman rank order correlation test provided additional evidence for the complex relationships between the examined genes. The statistically significant Spearman CC values $(p<0.0001)$ calculated for each pair of genes are depicted in Table II. The positive or negative relationships observed between the tested genes are in agreement with the above described observations.

Hierarchical clustering of gene expression was able to classify the cervical tissue samples according to disease status, with few discrepancies $(6.6 \%)$. Thus, this array of G1/S checkpoint markers could be used for diagnostic purposes. The examined genes were clustered in three groups of low, TP73 exon 2, RBL1 and TP73; moderate, POLA, CCND1, CDK2, E2F4, CDK6, MDM2, B2M, CDKN2D, MYC, CDKN1A, RBL2, E2F2, TP73 exon 13, CCNE1, and CDKN2A; and high, CDK4, E2F3, E2F5, E2F1, RB1 PCNA, CDKN1B, TP53 and DHFR, expression.

Previous studies have recognized the diagnostic significance of immunohistochemical analysis for CDKN2A alone (43-46) or in combination with other proteins, such as CDKN1A, CDKN1B, CCNE, CDKN2D and TP53 (47-50), for the early detection of reactive changes from LSIL to HSIL and cervical cancer. In this study, we identified CDKN1A as an early responsive gene to neoplasia.

In conclusion, our data outline important steps in carcinogenesis of the uterine cervix. Deregulation of G1/S phase transition takes place sequentially. Different genes are transcriptionally activated in low- and high-grade pre-cancerous lesions, and cancer. The first group of early responsive genes, comprised TP53, MDM2, E2F4, CDKN1A, CDKN2A and DHFR, are activated in LSIL. The second group, comprised E2F1, E2F3, E2F5, CCND1, CDK2, CDKN1B, PCNA and POLA, are activated in HSIL. The third group of late responsive or cancer-related genes comprised RBL2, E2F2, CDK6, CCNE1, TP73 and MYC, which are upregulated only in squamous cervical carcinomas. RBL1 was found to be downregulated in all three groups of cases and TP73 exhibited deregulation of isoform equilibrium. Hierarchical clustering 
can discriminate the cervical tissue biopsies according to disease status, which suggests a possible diagnostic importance.

\section{References}

1. Parkin DM, Bray F, Ferlay J and Pisani P: Global cancer statistics, 2002. CA Cancer J Clin 55: 74-108, 2005.

2. zur Hausen H: Papillomaviruses causing cancer: evasion from host-cell control in early events in carcinogenesis. J Natl Cancer Inst 92: 690-698, 2000.

3. Munoz N, Bosch FX, de Sanjose S, Herrero R, Castellsague X, Shah KV, Snijders PJ and Meijer CJ: Epidemiologic classification of human papillomavirus types associated with cervical cancer. N Engl J Med 348: 518-527, 2003.

4. Kirnbauer R, Booy F, Cheng N, Lowy DR and Schiller JT: Papillomavirus L1 major capsid protein self-assembles into virus-like particles that are highly immunogenic. Proc Natl Acad Sci USA 89: 12180-12184, 1992.

5. Rose RC, Bonnez W, Reichman RC and Garcea RL: Expression of human papillomavirus type $11 \mathrm{~L} 1$ protein in insect cells: in vivo and in vitro assembly of viruslike particles. J Virol 67: 1936-1944, 1993.

6. Kirnbauer R, Taub J, Greenstone H, Roden R, Durst M, Gissmann L, Lowy DR and Schiller JT: Efficient self-assembly of human papillomavirus type $16 \mathrm{~L} 1$ and L1-L2 into virus-like particles. J Virol 67: 6929-6936, 1993.

7. Skinner SR, Garland SM, Stanley MA, Pitts M and Quinn MA: Human papillomavirus vaccination for the prevention of cervical neoplasia: is it appropriate to vaccinate women older than 26? Med J Aust 188: 238-242, 2008.

8. Goodwin EC and DiMaio D: Repression of human papillomavirus oncogenes in HeLa cervical carcinoma cells causes the orderly reactivation of dormant tumor suppressor pathways. Proc Natl Acad Sci USA 97: 12513-12518, 2000.

9. Molden T, Kraus I, Karlsen F, Skomedal H, Nygard JF and Hagmar B: Comparison of human papillomavirus messenger RNA and DNA detection: a cross-sectional study of 4,136 women $>30$ years of age with a 2 -year follow-up of high-grade squamous intraepithelial lesion. Cancer Epidemiol Biomarkers Prev 14: 367-372, 2005

10. Schwarz E, Freese UK, Gissmann L, Mayer W, Roggenbuck B, Stremlau A and zur Hausen H: Structure and transcription of human papillomavirus sequences in cervical carcinoma cells. Nature 314: 111-114, 1985.

11. Klimov E, Vinokourova S, Moisjak E, Rakhmanaliev E, Kobseva V, Laimins L, Kisseljov F and Sulimova G: Human papilloma viruses and cervical tumours: mapping of integration sites and analysis of adjacent cellular sequences. BMC Cancer 2: 24,2002

12. Jeon $\mathrm{S}$ and Lambert PF: Integration of human papillomavirus type 16 DNA into the human genome leads to increased stability of E6 and E7 mRNAs: implications for cervical carcinogenesis. Proc Natl Acad Sci USA 92: 1654-1658, 1995

13. Jeon S, Allen-Hoffmann BL and Lambert PF: Integration of human papillomavirus type 16 into the human genome correlates with a selective growth advantage of cells. J Virol 69: 2989-2997, 1995

14. Dyson N, Howley PM, Munger K and Harlow E: The human papilloma virus-16 E7 oncoprotein is able to bind to the retinoblastoma gene product. Science 243: 934-937, 1989.

15. Munger K, Werness BA, Dyson N, Phelps WC, Harlow E and Howley PM: Complex formation of human papillomavirus E7 proteins with the retinoblastoma tumor suppressor gene product. EMBO J 8: 4099-4105, 1989.

16. Werness BA, Levine AJ and Howley PM: Association of human papillomavirus types 16 and 18 E6 proteins with p53. Science 248: 76-79, 1990

17. Deshpande A, Sicinski $\mathrm{P}$ and Hinds PW: Cyclins and cdks in development and cancer: a perspective. Oncogene 24: 2909-2915, 2005.

18. Sherr CJ: Mammalian G1 cyclins and cell cycle progression. Proc Assoc Am Physicians 107: 181-186, 1995.

19. Stevens C and La Thangue NB: E2F and cell cycle control: a double-edged sword. Arch Biochem Biophys 412: 157-169, 2003.

20. Cobrinik D: Pocket proteins and cell cycle control. Oncogene 24: 2796-2809, 2005.
21. Cam H and Dynlacht BD: Emerging roles for E2F: beyond the G1/S transition and DNA replication. Cancer Cell 3: 311-316, 2003.

22. Dimova DK and Dyson NJ: The E2F transcriptional network: old acquaintances with new faces. Oncogene 24: 2810-2826, 2005.

23. Harris SL and Levine AJ: The p53 pathway: positive and negative feedback loops. Oncogene 24: 2899-2908, 2005.

24. Lowe SW and Sherr CJ: Tumor suppression by Ink4a-Arf: progress and puzzles. Curr Opin Genet Dev 13: 77-83, 2003.

25. Marin MC and Kaelin WG Jr: p63 and p73: old members of a new family. Biochim Biophys Acta 1470: M93-M100, 2000.

26. Lissy NA, Davis PK, Irwin M, Kaelin WG and Dowdy SF: A common E2F-1 and p73 pathway mediates cell death induced by TCR activation. Nature 407: 642-645, 2000.

27. Yamasaki L: Role of the RB tumor suppressor in cancer. Cancer Treat Res 115: 209-239, 2003.

28. Castle PE, Wacholder S, Lorincz AT, Scott DR, Sherman ME, Glass AG, Rush BB, Schussler JE and Schiffman M: A prospective study of high-grade cervical neoplasia risk among human papillomavirus-infected women. J Natl Cancer Inst 94: 1406-1414, 2002.

29. Ferenczy A and Franco E: Persistent human papillomavirus infection and cervical neoplasia. Lancet Oncol 3: 11-16, 2002.

30. de Roda Husman AM, Walboomers JM, van den Brule AJ, Meijer CJ and Snijders PJ: The use of general primers GP5 and GP6 elongated at their 3' ends with adjacent highly conserved sequences improves human papillomavirus detection by PCR. J Gen Virol 76: 1057-1062, 1995.

31. Arends MJ, Donaldson YK, Duvall E, Wyllie AH and Bird CC: HPV in full thickness cervical biopsies: high prevalence in CIN 2 and CIN 3 detected by a sensitive PCR method. J Pathol 165: 301-309, 1991.

32. Chen KY: Transcription factors and the down-regulation of G1/S boundary genes in human diploid fibroblasts during senescence. Front Biosci 2: D417-D426, 1997.

33. Farkas T, Hansen K, Holm K, Lukas J and Bartek J: Distinct phosphorylation events regulate p130- and p107-mediated repression of E2F-4. J Biol Chem 277: 26741-26752, 2002.

34. Parakati R and Dimario JX: Dynamic transcriptional regulatory complexes including E2F4, p107, p130, and Sp1 control fibroblast growth factor receptor 1 gene expression during myogenesis. J Biol Chem 280: 21284-21294, 2005.

35. Crosby ME and Almasan A: Opposing roles of E2Fs in cell proliferation and death. Cancer Biol Ther 3: 1208-1211, 2004.

36. La Thangue NB: The yin and yang of E2F-1: balancing life and death. Nat Cell Biol 5: 587-589, 2003.

37. Tashiro E, Maruki H, Minato Y, Doki Y, Weinstein IB and Imoto M: Overexpression of cyclin D1 contributes to malignancy by up-regulation of fibroblast growth factor receptor 1 via the pRB/E2F pathway. Cancer Res 63: 424-431, 2003.

38. Lee RJ, Albanese C, Fu M, D'Amico M, Lin B, Watanabe G, Haines GK III, Siegel PM, Hung MC, Yarden Y, Horowitz JM, Muller WJ and Pestell RG: Cyclin D1 is required for transformation by activated Neu and is induced through an E2Fdependent signaling pathway. Mol Cell Biol 20: 672-683, 2000.

39. Hwang HC and Clurman BE: Cyclin E in normal and neoplastic cell cycles. Oncogene 24: 2776-2786, 2005.

40. Woo RA and Poon RY: Cyclin-dependent kinases and S phase control in mammalian cells. Cell Cycle 2: 316-324, 2003.

41. Arvanitis DA, Lianos E, Soulitzis N, Delakas D and Spandidos DA: Deregulation of p73 isoform equilibrium in benign prostate hyperplasia and prostate cancer. Oncol Rep 12: 1131-1137, 2004

42. Harms KL and Chen $X$ : The $C$ terminus of p53 family proteins is a cell fate determinant. Mol Cell Biol 25: 2014-2030, 2005.

43. Nieh S, Chen SF, Chu TY, Lai HC, Lin YS, Fu E and Gau CH: Is p16(INK4A) expression more useful than human papillomavirus test to determine the outcome of atypical squamous cells of undetermined significance-categorized Pap smear? A comparative analysis using abnormal cervical smears with follow-up biopsies. Gynecol Oncol 97: 35-40, 2005.

44. Murphy N, Ring M, Heffron CC, King B, Killalea AG, Hughes C, Martin CM, McGuinness E, Sheils O and O'Leary JJ: p16INK4A, CDC6, and MCM5: predictive biomarkers in cervical preinvasive neoplasia and cervical cancer. J Clin Pathol 58: 525-534, 2005.

45. Volgareva G, Zavalishina L, Andreeva Y, Frank G, Krutikova E, Golovina D, Bliev A, Spitkovsky D, Ermilova V and Kisseljov F: Protein p16 as a marker of dysplastic and neoplastic alterations in cervical epithelial cells. BMC Cancer 4: 58, 2004. 
46. Santin AD, Zhan F, Bignotti E, Siegel ER, Cane S, Bellone S, Palmieri M, Anfossi S, Thomas M, Burnett A, Kay HH, Roman JJ, O'Brien TJ, Tian E, Cannon MJ, Shaughnessy J Jr and Pecorelli S: Gene expression profiles of primary HPV16and HPV18-infected early stage cervical cancers and normal cervical epithelium: identification of novel candidate molecular markers for cervical cancer diagnosis and therapy. Virology 331: 269-291, 2005.

47. Wang JL, Zheng BY, Li XD, Angstrom T, Lindstrom MS and Wallin KL: Predictive significance of the alterations of p16INK4A, p14ARF, p53, and proliferating cell nuclear antigen expression in the progression of cervical cancer. Clin Cancer Res 10: 2407-2414, 2004.

48. Moore GD, Lear SC, Wills-Frank LA, Martin AW, Snyder JW and Helm CW: Differential expression of cdk inhibitors p16, p21cip1, p27kip1, and cyclin E in cervical cytological smears prepared by the ThinPrep method. Diagn Cytopathol 32: 82-87, 2005.
49. Keating JT, Cviko A, Riethdorf S, Riethdorf L, Quade BJ, Sun D, Duensing S, Sheets EE, Munger K and Crum CP: Ki-67, cyclin $\mathrm{E}$, and p16INK4 are complimentary surrogate biomarkers for human papilloma virus-related cervical neoplasia. Am J Surg Pathol 25: 884-891, 2001.

50. Wang JL, Zheng BY, Li XD, Nokelainen K, Angstrom T, Lindstrom MS and Wallin KL: p16INK4A and p14ARF expression pattern by immunohistochemistry in human papillomavirus-related cervical neoplasia. Mod Pathol 18: 629-637, 2005. 